OPEN ACCESS

Edited by:

Joao Inacio,

University of Brighton,

United Kingdom

Reviewed by:

Xia Xiong,

Institute of Subtropical Agriculture,

Chinese Academy of Sciences, China

Seungha Kang,

University of Queensland, Australia

Wenkai Ren,

South China Agricultural University,

China

${ }^{*}$ Correspondence: Zhiyong Fan

fzyong04@163.com

Specialty section:

This article was submitted to

Systems Microbiology,

a section of the journal

Frontiers in Microbiology

Received: 03 August 2020 Accepted: 11 December 2020 Published: 08 January 2021

Citation:

Zhang LL, Gu XL, Wang J, Liao S,

Duan YH, Li H, Song ZH, He X and

Fan ZY (2021) Effects of Dietary

Isomaltooligosaccharide Levels on

the Gut Microbiota, Immune Function

of Sows, and the Diarrhea Rate

of Their Offspring.

Front. Microbiol. 11:588986

doi: $10.3389 /$ fmich.2020.588986

\section{Effects of Dietary}

Isomaltooligosaccharide Levels on the Gut Microbiota, Immune Function of Sows, and the Diarrhea Rate of Their Offspring

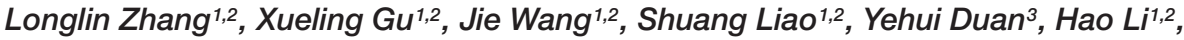 \\ Zehe Song ${ }^{1,2}, \mathrm{Xi} \mathrm{He}^{1,2}$ and Zhiyong Fan ${ }^{1,2 *}$
}

\begin{abstract}
${ }^{1}$ College of Animal Science and Technology, Hunan Agricultural University, Changsha, China, ${ }^{2}$ Hunan Co-Innovation Center of Animal Production Safety, Hunan Agricultural University, Changsha, China, ${ }^{3}$ Hunan Provincial Key Laboratory of Animal

Nutritional Physiology and Metabolic Process, Key Laboratory of Agro-ecological Processes in Subtropical Region, Institute of Subtropical Agriculture, Chinese Academy of Sciences, Hunan Provincial Engineering Research Center for Healthy Livestock and Poultry Production, Scientific Observing and Experimental Station of Animal Nutrition and Feed Science in South-Central, Ministry of Agriculture, National Engineering Laboratory for Pollution Control and Waste Utilization in Livestock and Poultry Production, Changsha, China
\end{abstract}

To investigate the effects of dietary isomaltooligosaccharide (IMO) levels on the gut microbiota, immune function of sows, and the diarrhea rate of their offspring, 120 multiparous gestating pig improvement company (PIC) sows with similar body conditions were selected and fed 1 of 6 diets: a basal diet with no supplement (control, CON), or a diet supplemented with $2.5 \mathrm{~g} / \mathrm{kg}, 5.0 \mathrm{~g} / \mathrm{kg}, 10.0 \mathrm{~g} / \mathrm{kg}, 20.0 \mathrm{~g} / \mathrm{kg}$, or $40.0 \mathrm{~g} / \mathrm{kg}$ IMO (IMO1, IMO2, IMO3, IMO4, or IMO5 group, respectively). Results showed that dietary treatments did not affect the reproductive performance and colostrum composition of sows $(P>0.05)$. However, compared to the CON, IMO reduced the diarrhea rate of suckling piglets $(P<0.05)$ and improved the concentrations of colostrum IgA, IgG, and IgM $(P<0.05)$. Moreover, IMO decreased the concentrations of serum D-lactate (D-LA) and lipopolysaccharides (LPS) at farrowing and day 18 of lactation (L18) $(P<0.05)$. High-throughput pyrosequencing of the $16 \mathrm{~S}$ rRNA demonstrated that IMO shaped the composition of gut microbiota in different reproductive stages (day 107 of gestation, G107; day 10 of lactation, $L 10)(P<0.05)$. At the genus level, the relative abundance of g_Parabacteroides and g_Slackia in G107 and g_Unclassified_Peptostreptococcaceae, g_Turicibacter, g_Sarcina, and g_Coprococcus in L10 was increased in IMO groups but the g_YRC22 in G107 was decreased in IMO groups relative to the CON group $(P<0.05)$. Furthermore, the serum D-LA and LPS were negatively correlated with the genus g_Akkermansia and g_Parabacteroides but positively correlated with the genus g_YRC22 and g_Unclassified_Peptostreptococcaceae. Additionally, the colostrum IgA, IgG, and IgM of sows were positively correlated with the genus g_Parabacteroides, g_Sarcina, and 
g_Coprococcus but negatively correlated with the genus g_YRC22. These findings indicated that IMO could promote the immune activation and had a significant influence in sows' gut microbiota during perinatal period, which may reduce the diarrhea rate of their offspring.

Keywords: reproductive performance, diarrhea, gut microbiota, sows, isomaltooligosaccharide

\section{INTRODUCTION}

The perinatal period of sows is the transition stage from gestation to lactation, which generally refers to the combined period of late gestation and early lactation (10 days before after delivery). And sows are the core of modern large-scale pig production; their health and reproductive performance are the key to the economic benefits of pig farms. There were massive researches which suggest that the gut microbiota homeostasis of sow is important for the healthy gut development of their offspring during perinatal period (Zambrano et al., 2016; Grześkowiak et al., 2020). In addition, the physiological condition of suckling piglets is closely connected with the sows in the first weeks of life, including energy and nutrient supply (Klobasa et al., 1987; Gao et al., 2020), immunological protection (Salmon et al., 2009) and the microbial colonization of the gastrointestinal tract. So far, there are increasingly studies on the sow-piglet-axis, but there are not many systematic research literatures in this field.

Previous studies have demonstrated that a probiotic treatment of sows altered the composition of the gut microbiota in their offspring (Mori et al., 2011; Baker et al., 2013; Starke et al., 2013). Isomaltooligosaccharides (IMO), as a functional oligosaccharide, is considered to act as a prebiotic, since it can modulate the composition and metabolic activity of the gut microbiota, which might potentially enhance the health of the host organism (Ketabi et al., 2011). In particular, previous studies have shown that Bifidobacterium and Lactobacillus were increased in fecal microbiota, when different doses of IMO were supplemented to the diets (Ketabi et al., 2011; Yen et al., 2011; Likotrafiti et al., 2014). Besides, IMO is also known for its potential to activate the immune system and to enhance the host's resistance to diseases and oxidation ( $\mathrm{Wu}$ et al., 2017). However, no studies have been conducted to evaluate the effects of dietary IMO in gestating and lactating sows, although dietary IMO supplementation directly improved immune status and diarrhea of piglets (Wang et al., 2016; Wu et al., 2017). This aspect might be interesting, as a modulation of the gut microbiota of the sows might also influence the structure and composition of bacteria in the intestinal tract of their piglets. In addition, few studies have evaluated the potential of prebiotics in the sow-piglet-axis up to now, particular IMO. Therefore, it is necessary to study on the mechanism of IMO supplementation with respect to sow-piglet-axis.

Consequently, the purpose of this study was to study the effects of dietary IMO levels on reproductive performance, colostrum composition, immune index and gut microbiota of sows and diarrhea of offspring.

\section{MATERIALS AND METHODS}

\section{Animal, Diets and Experimental Design}

The protocol of this study was approved by the Institution Animal Care and Use Committee of college of Animal Science and Technology, Hunan Agricultural University (Changsha, China), and was conducted in accordance with the National Institutes of Health (Changsha, China) guidelines for the care and use of experimental animals. The IMO (IMO-900; purity $\geq 90 \%$, with total isomaltose, panose, and isomaltotriose contents > 45\%) was provided by the Baolingbao Biology Company (Shandong, China).

One hundred and twenty late pregnant sows (day 85 of gestation, PIC) with an initial body weight of $253.36 \pm 14.30 \mathrm{~kg}$ and parity of $5.27 \pm 1.58$ were randomly allocated to 1 of 6 dietary treatments with 20 replicates based on body weight, parity and back fat. The treatments are: 1) a basal diet from late gestation to farrowing (CON group), 2) a basal diet plus $2.5 \mathrm{~g} / \mathrm{kg}, 5.0 \mathrm{~g} / \mathrm{kg}, 10.0 \mathrm{~g} / \mathrm{kg}, 20.0 \mathrm{~g} / \mathrm{kg}$ or $40.0 \mathrm{~g} / \mathrm{kg}$ IMO (IMO1, IMO2, IMO3, IMO4, or IMO5 groups). The composition of basal diets (Supplementary Table 1) was formulated in compliance with NRC (1998) nutrient requirements.

Sows were housed in $2.0 \mathrm{~m} \times 0.6 \mathrm{~m}$ concrete-floored farrowing pen during day 85 to day 107 of gestation. The average amount fed to sows in each group was half provided at each feeding for a total of $2.6-2.8 \mathrm{~kg}$, twice a day $(08: 00$ am and 15:00 pm). During gestation day 108 to lactation, the sows were housed indoors in $2.13 \mathrm{~m} \times 0.66 \mathrm{~m}$ concrete-floored delivery room pen. The average amount fed to sows in each group was half provided at each feeding for a total of $3.2 \mathrm{~kg}$, twice a day $(08: 00 \mathrm{am}$ and $16: 30 \mathrm{pm})$. Before farrowing days $1-2$, the average feeding amount dropped to $2.0 \mathrm{~kg} \mathrm{~d}^{-1}$. On the farrowing day (day 0 of lactation), the sows initially received a total of $1.0 \mathrm{~kg} \mathrm{day}^{-1}$ of their lactation diets, which was then increased by $0.8 \mathrm{~kg} \mathrm{day}{ }^{-1}$ on days 1 and 2 and by $1.0 \mathrm{~kg} \mathrm{day}^{-1}$ on days 3 and 4 until arriving at their maximum feed intake. To feed sows diets ad libitum to ensure that the sow's trough has surplus fodder from day 5 of lactation to weaning. Sows were provided ad libitum access to water during the experimental period. The experiment was carried out in Hunan Xinguangan Agriculture and Animal Husbandry Co., Ltd. Pingjiang Branch (Xinguangan, Inc., Hunan, China), and the feeding management and immunization procedure were carried out in accordance 
with the company's standard of breeding management. The trial lasted for 60 days.

\section{Sample Collection}

Seven sows per group were randomly selected for sample collection. Fresh feces were collected directly by massaging the rectum of sow on G107 and L10. Then, 60 samples stored in dry ice were transported to the laboratory and then stored at $80^{\circ} \mathrm{C}$ until analysis. A $10-\mathrm{mL}$ blood sample of sow from the ear vein was collected on farrowing day within $2 \mathrm{~h}$ after delivery and L18 after an overnight fasting period of 16-18 h. Serum samples were obtained by centrifuging at $3,000 \times g$ for $15 \mathrm{~min}$ at $4^{\circ} \mathrm{C}$ after standing for $1 \mathrm{~h}$ at $4^{\circ} \mathrm{C}$. Then the samples were immediately stored at $-80^{\circ} \mathrm{C}$ for the next analysis. Within two hours after farrowing, seven sows in each group were randomly selected for milk sample collection by hand-milking of four to six teats.

\section{Reproductive Performance and Diarrhea Rate of Piglets}

The total number of born, born alive, born robust (weight greater than $800 \mathrm{~g}$ ), stillborn, and mummy number was recorded, so were average piglet birth weight (BW) at farrowing and lactation as well as average daily feed intake (ADFI) during lactation. On this basis, the survival rate of piglets, litter weight gain (from day 3 after birth to weaning) and total milk yield [(weaning litter weight-initial litter weight)/4] were calculated. In addition, from birth to weaning, the fecal score of piglets of each litter was recorded daily and their diarrhea rate during days 1-3, 1-7, 1-14, and 1-21 after birth were also be calculated.

\section{Sow's Milk Composition and Immunity, and Serum D-Lactate and Lipopolysaccharides}

The milk samples of sows in each group were separately analyzed for the concentrations of fat, protein, lactose, urea nitrogen, fatting dry matter, and total dry matter using a Milko-Scan FT 120 (Foss Electric, Hillerford, Denmark). Colostrum and serum concentrations of immunoglobulin G (IgG), immunoglobulin A ( $\operatorname{IgA})$, and immunoglobulin $\mathrm{M}(\operatorname{Ig} M)$ were determined by the radial immunodiffusion method using a commercial kit (Wuhan Biological Engineering Co., Ltd, Wuhan, China). Besides, the serum D-lactate (D-LA) and lipopolysaccharides (LPS) were assessed by ELISA using commercially available kits (Wuhan Biological Engineering Co., Ltd, Wuhan, China).

\section{DNA Extraction 16S rDNA Amplification and 16S rRNA Sequencing}

DNA was extracted from fecal samples of sows (G107 and L10) using a Stool DNA Isolation Kit (Tiangen Biotech Co., Ltd., Beijing, China). The V3-V4 hypervariable region of the bacterial $16 \mathrm{~S}$ rRNA gene was amplified using universal primers $(338 \mathrm{~F}$ and $806 \mathrm{R}$ ). For each fecal sample, a 10-digit barcode sequence was added to the 5' end of the forward and reverse primers (provided by Allwegene Company, Beijing, China). The PCR components contained $5 \mu \mathrm{L}$ of Q 5 reaction buffer $(5 \times), 5 \mu \mathrm{L}$ of Q5 High-Fidelity GC buffer ( $5 \times), 0.25 \mu \mathrm{L}$ of Q5 high-fidelity

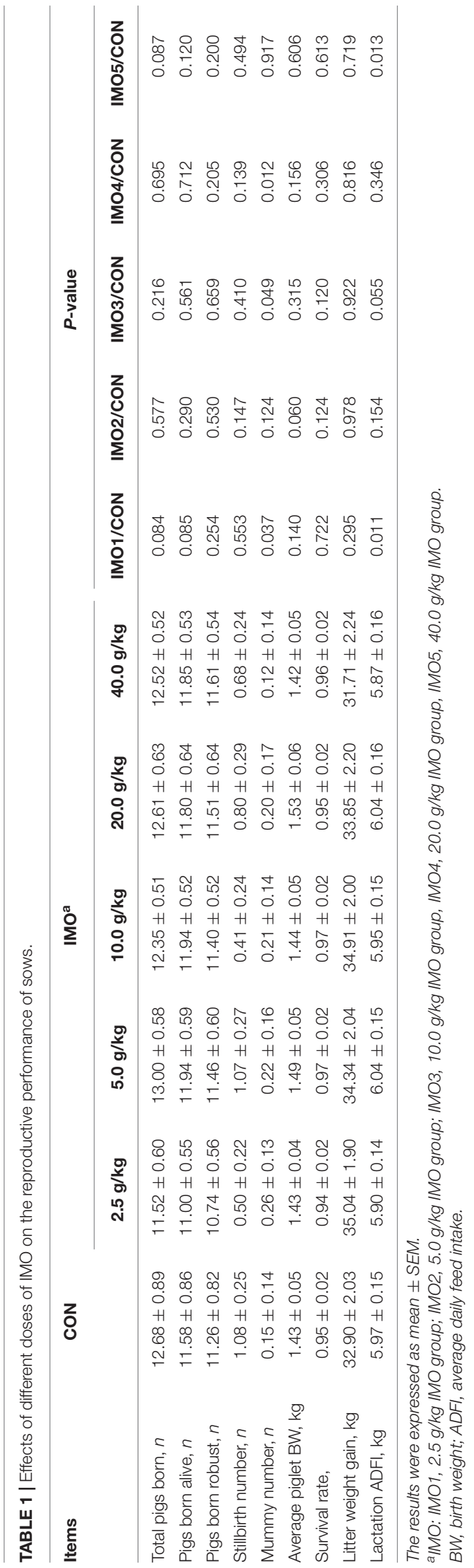


DNA polymerase $(5 \mathrm{U} / \mu \mathrm{L}), 2 \mu \mathrm{L}(2.5 \mathrm{mM})$ of dNTPs, $1 \mu \mathrm{L}$ $(10 \mathrm{uM})$ of each forward and reverse primer, $1 \mu \mathrm{L}$ of DNA template, and $9.75 \mu \mathrm{L}$ of $\mathrm{ddH}_{2} \mathrm{O}$. Cycling parameters were $98^{\circ} \mathrm{C}$ for $5 \mathrm{~min}$, followed by 25 cycles at $98^{\circ} \mathrm{C}$ for $30 \mathrm{~s}, 52^{\circ} \mathrm{C}$ for $30 \mathrm{~s}$, and $72^{\circ} \mathrm{C}$ for $1 \mathrm{~min}$, and a final extension at $72^{\circ} \mathrm{C}$ for 5 min. PCR amplicons were purified with Agencourt AMPure beads (Beckman Coulter, Indianapolis, IN, United States) and quantified using the PicoGreen dsDNA Assay Kit (Invitrogen, Carlsbad, CA, United States). After the individual quantification step, amplicons were pooled in equal amounts, and pair-end $2 \times 300$ bp sequencing was performed using the Illlumina MiSeq platform with MiSeq Reagent Kit v3 at Shanghai Personal Biotechnology Co., Ltd (Shanghai, China). The sequences were clustered into operational taxonomic units (OTUs) at a similarity level of $97 \%$ to generate rarefaction curves and to calculate the richness and diversity indices. OTUs representing $<0.005 \%$ of the population were removed and taxonomy was assigned by the Ribosomal Database Project (RDP) classifier.

The Qiime ${ }^{1}$ software (Caporaso et al., 2010) was employed to process the sequencing data and perform cluster analysis,

${ }^{1}$ V1.8.0, http://qiime.org/
ACE abundance indexing, and Simpson diversity indexing of the analysis results. In addition, USEARCH ${ }^{2}$ was used to exclude chimeric sequences. Beta diversity analysis was used to investigate the structural variation of microbial communities across samples using UniFrac distance metrics. The Spearman's rho nonparametric correlations between the gut microbiota and immune-related indexes were determined using R packages (v3.5.2).

\section{Statistical Analysis}

An individual sow served as the experimental unit. All statistical analyses were performed using SPSS 20.0 software (SPSS Inc., Chicago, IL, United States). The differences among groups were compared using covariance analysis, one-way ANOVA and Duncan multiple range test. In addition, the reproductive performance and colostrum composition of sows were compared with use of independent-samples $T$-test, and the diarrhea rates of suckling piglets were compared with use of chi-square analysis. Significance was set at $P<0.05$.

${ }^{2}$ v5.2.236, http://www.drive5.com/usearch/

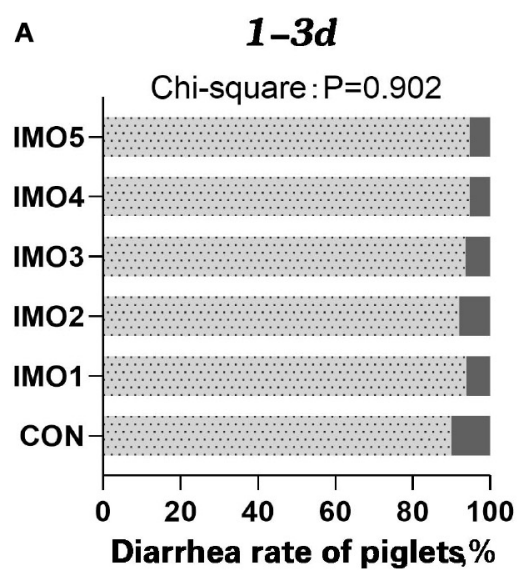

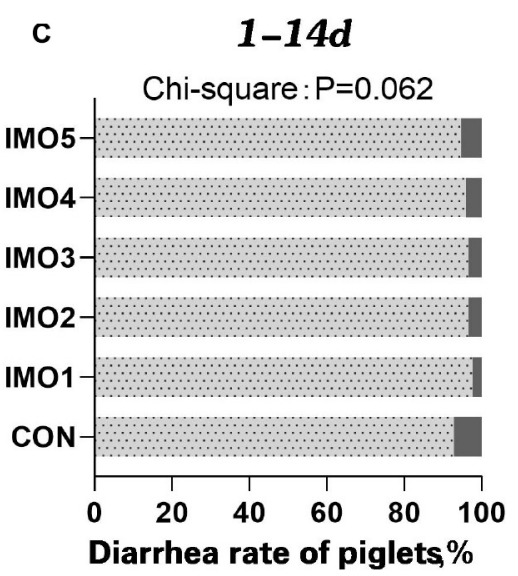

Diarrhea

No diarrhea
B

1-7d

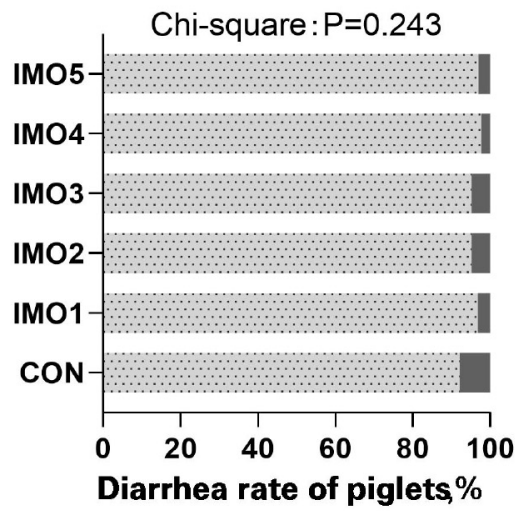

D

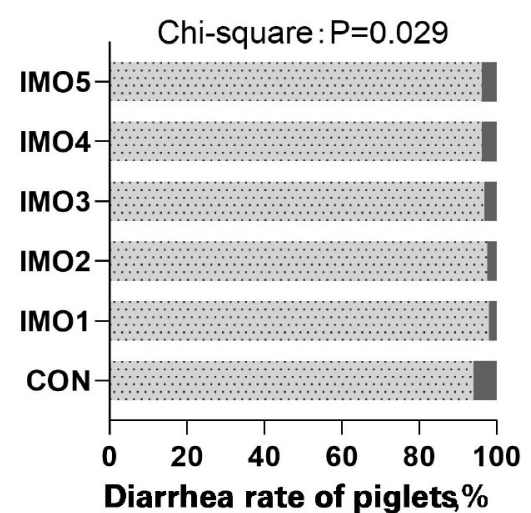

Diarrhea

No diarrhea

FIGURE 1 | Effects of different doses of IMO on the diarrhea of suckling piglets in a different stage [(A), 1-3 days; (B) 1-7 days; (C) 1-14 days; (D) 1-21 days]. Data were compared with use of chi-square analysis $(P<0.10)$. 


\section{RESULTS}

\section{Reproductive Performance on Sows}

The effects of dietary IMO levels on reproductive performance of sows were shown in Table 1. There were also no effects of IMO treatment on mainly reproductive performance of sows $(P>0.05)$, in addition to mummy number and lactation ADFI $(P<0.05)$.

\section{The Diarrhea Rate of Suckling Piglets}

The results of dietary IMO levels on diarrhea rate of piglets were shown in Figure 1. The diarrhea rate of piglets was awfully high at days 1 to 14 of lactation (1-14 days) and days 1 to 21 of lactation (1-21 days), whereas dietary IMO reversed the diarrhea rate of piglets in a dose-dependent manner $(P<0.05$, Figures 1C,D).

\section{The Colostrum Composition and Immunity of Sows}

Effects of dietary IMO levels on colostrum composition and immunity were shown in Table 2 and Figure 2. The results displayed that dietary IMO had no marked effect on the colostrum composition of sows $(P>0.05$, Table 2$)$. However, sows fed the IMO had a higher IgA, IgG, and IgM concentration in colostrum, with the highest values observed in IMO3 and IMO4 groups $(P<0.001$, Figure 2$)$.

\section{The Serum Immunity of Sows}

As shown in Figure 3, sows in the IMO5 group exhibited the highest concentrations of serum IgA, IgG, and IgM $(P<0.05)$ at farrowing. Besides, the IMO4 significantly increased the serum IgG concentration compared with $\mathrm{CON}$, IMO1, and IMO2 groups $(P<0.05$, Figure 3B).

\section{The Serum Biomarker of Sows}

At farrowing, the D-LA concentration in IMO1 group and LPS concentration in IMO1, IMO3, IMO4 and IMO5 groups were lower than those in the CON group (Linear, $P<0.05$, Figures 4 A,C). In addition, sows fed the diets containing IMO5 had a higher concentration of serum D-LA compared to IMO1 and IMO2 in L18 (Linear, $P<0.05$ ), but the lower concentration of serum LPS was shown in IMO4 and IMO5 groups (Linear, $P<0.05$, Figures 4B,D).

\section{The Gut Microbiota Diversity and Composition of Sows}

Isomaltooligosaccharide changed the gut microbiota diversity of sows in G107 and L10 but the trend was different. The Chao 1 and ACE diversity indices were significantly reduced by dietary IMO supplementation in sows (expect IMO1 group) compared with the CON group in G107 (Linear, $P<0.05$, Figure 5A). The Simpson and Shannon diversity indices were increased in all IMO groups, and the significantly difference was observed among the CON group and IMO3 and IMO4 groups in L10 (Quadratic, $P<0.05$, Figure 5B).

All 60 fecal samples were subjected to 16S rRNA gene sequencing. Illumina Miseq sequencing of the V3-V4

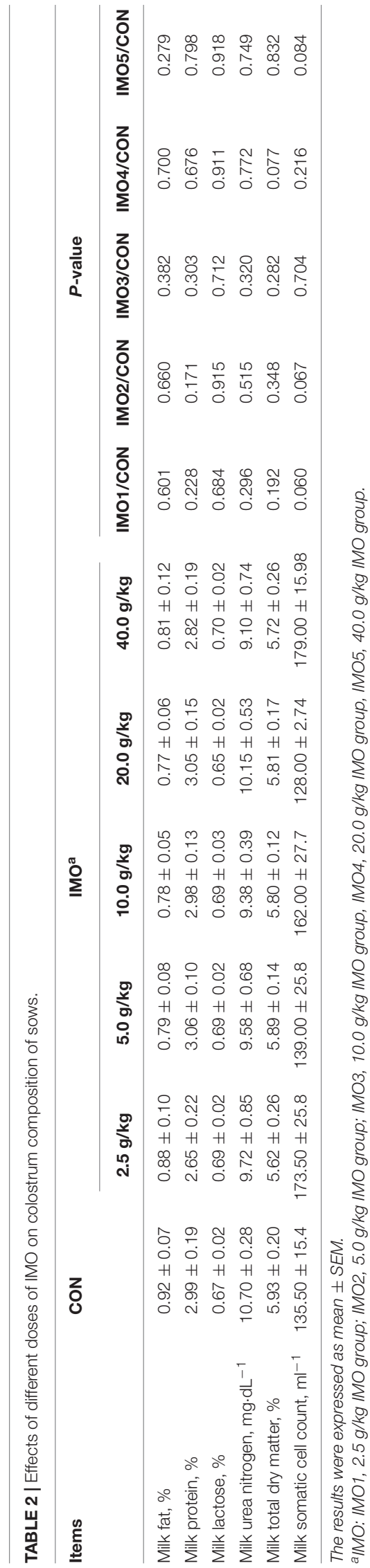



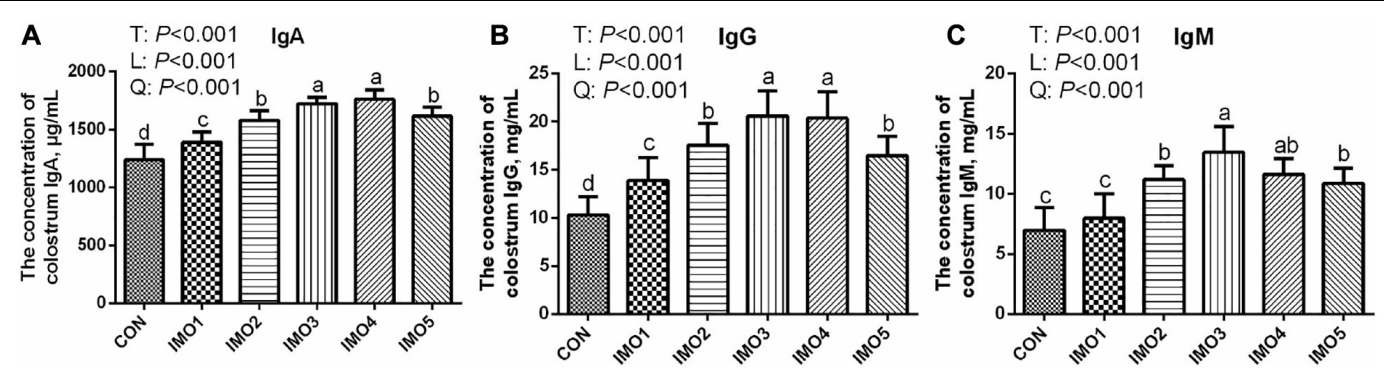

FIGURE 2 | Effects of dietary isomaltooligosaccharide (IMO) levels on the concentrations of colostrum IgA (A), IgG (B), and IgM (C). Data are presented as means $\pm \mathrm{SD}(n=7)$. (a-d) Significant effects of treatment $(P<0.05$; values with different lowercase letters are significantly different; $T$, total; $L$, linear; $Q$, quadratic).
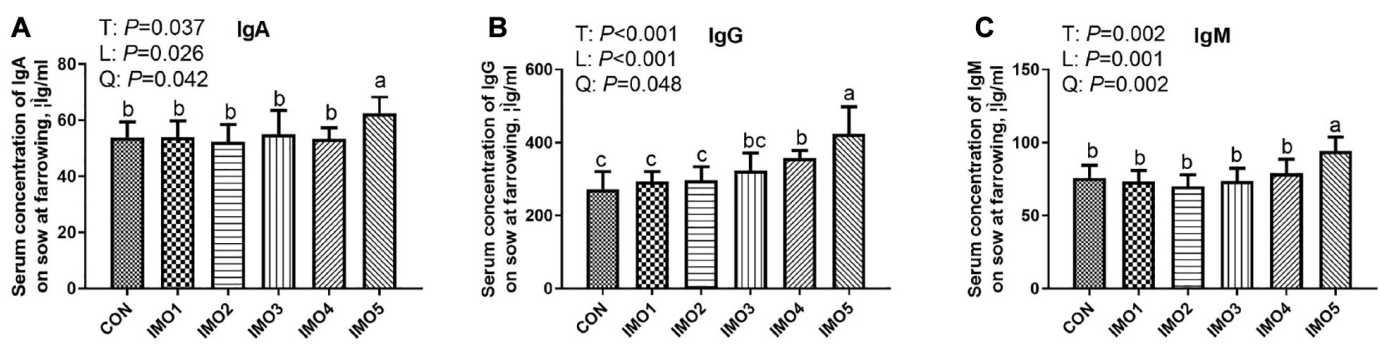

FIGURE 3 | Effects of dietary isomaltooligosaccharide (IMO) levels on the concentrations of serum IgA (A), IgG (B), and IgM (C). Data are presented as means \pm SD $(n=7)$. $(\mathrm{a}-\mathrm{C})$ Significant effect of treatment $(P<0.05$; values with different lowercase letters are significantly different; $T$, total; $L$, linear; $Q$, quadratic).

regions of bacterial $16 \mathrm{~S}$ rRNA genes generated 1,094,499 and $1,132,136$ high-quality sequences in G107 and L10, respectively (Supplementary Table 2). On the basis of $97 \%$ sequence similarity, we obtained 7,806 and 7,969 OTUs in G107 and L10, respectively. Further, variations in the microbial composition of all groups were explored. LEfSe analysis of the bacterial community was used to filter the significantly different OTUs among groups and the results showed that there exist dramatic differences in microbial composition between the treatment groups and the CON group (Supplementary Figure 1). There were no significant difference in the relative abundance of bacterial p_Firmicutes and p_Bacteroidetes and its ratio (F/B) among the all treatment groups in G107 and L10 $(P>0.05$, Figures 6A-C, 7A,B). g_Lactobacillus increased but g_YRC22 showed a remarkable reduction in IMO4 group compared with the CON group in G107 (Linear, $P<0.05$, Figures 6D,E). Sows in IMO3 group had a highest relative abundance of g_Unclassified_Coriobacteriaceae and g_Slackia, and it significantly differ from IMO2, IMO4, and IMO5 groups in the relative abundance of g_Unclassified_Coriobacteriaceae and CON group, IMO1,IMO2, IMO4, and IMO5 groups in the relative abundance of g_Slackia (Total, $P<0.05$, Figures 6F,H). Besides, the relative abundance of $g_{-}$Parabacteroides was enhanced in IMO2 and IMO3 groups (Quadratic, $P<0.05$, Figure 6G) and the relative abundance of g_Bifidobacterium was increased in IMO4 and IMO5 groups $(P>0.05$, Figure 6I). At the genus level, the relative abundances of g_Unclassified_Ruminococcaceae and g_Coprococcus were the highest (Total, $P<0.05$, Figures 7C,E) but the g_Unclassified_Peptostreptococcaceae and g_Sarcina was the lowest in IMO4 group in L10 $(P<0.05$,
Figures 7D,G). In addition, the relative abundances of g_Unclassified_Peptostreptococcaceae, g_Turicibacter and g_Sarcina were the highest in IMO2 group (Quadratic, $P<0.05$, Figures $7 \mathbf{D}, \mathbf{F}, \mathbf{G})$ and the relative abundance of g_Akkermansia was the highest in IMO1 group $(P>0.05$, Figure $7 \mathbf{H})$. Furthermore, IMO increased the relative abundance of g_Bifidobacterium compared with the CON $(P>0.05$, Figure 7I). Therefore, the above results indicated that the gut microbiota composition of sows was profoundly altered during late pregnancy and lactation.

\section{Correlations Between Gut Microbiota and Serum Biomarker and Colostric Immunoglobulin}

A Spearman's correlation analysis was performed to evaluate the potential link between alterations in gut microbiota composition in G107 and L10 and serum D-LA, LPS, IgA, IgG, and IgM of sows at farrowing and $\mathrm{L} 18$ or $\operatorname{IgA}$, IgG, and IgM in colostrum of sows at farrowing (Figure 8). The serum D-LA and LPS at farrowing was negatively correlated with the genus g_Akkermansia and g_Parabacteroides in L10 $(P<0.05)$, respectively. However, the serum LPS in L18 was positively correlated with the genus g_YRC22 in G107 and the genus g_Unclassified_Peptostreptococcaceae in L10 $(P<0.05)$. Moreover, the IgA, IgG, and IgM in colostrum of sows at farrowing was positively correlated with the genus g_Parabacteroides and g_Slackia in G107 and g_Coprococcus in L10 but negatively correlated with the genus g_YRC22 in G107 $(P<0.05)$. 

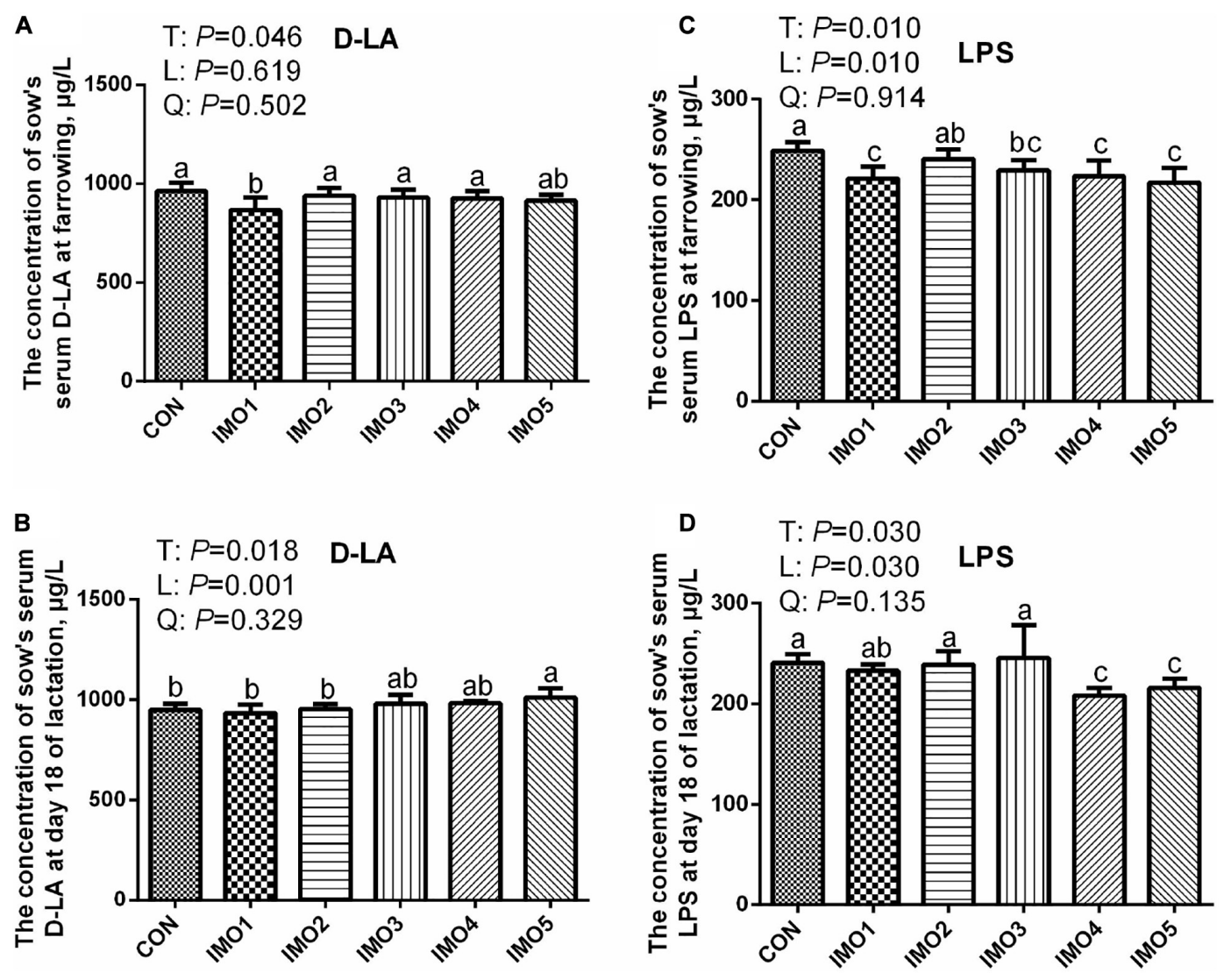

FIGURE 4 | Effects of dietary isomaltooligosaccharide (IMO) levels on the concentrations of sows' serum D-LA (A) and LPS (C) at farrowing as well as serum D-LA (B) and LPS (D) at day 18 of lactation. Data are presented as means $\pm \mathrm{SD}(n=7)$. (a-c) Significant effect of treatment $(P<0.05$; values with different lowercase letters are significantly different; $T$, total; L, linear; $Q$, quadratic).

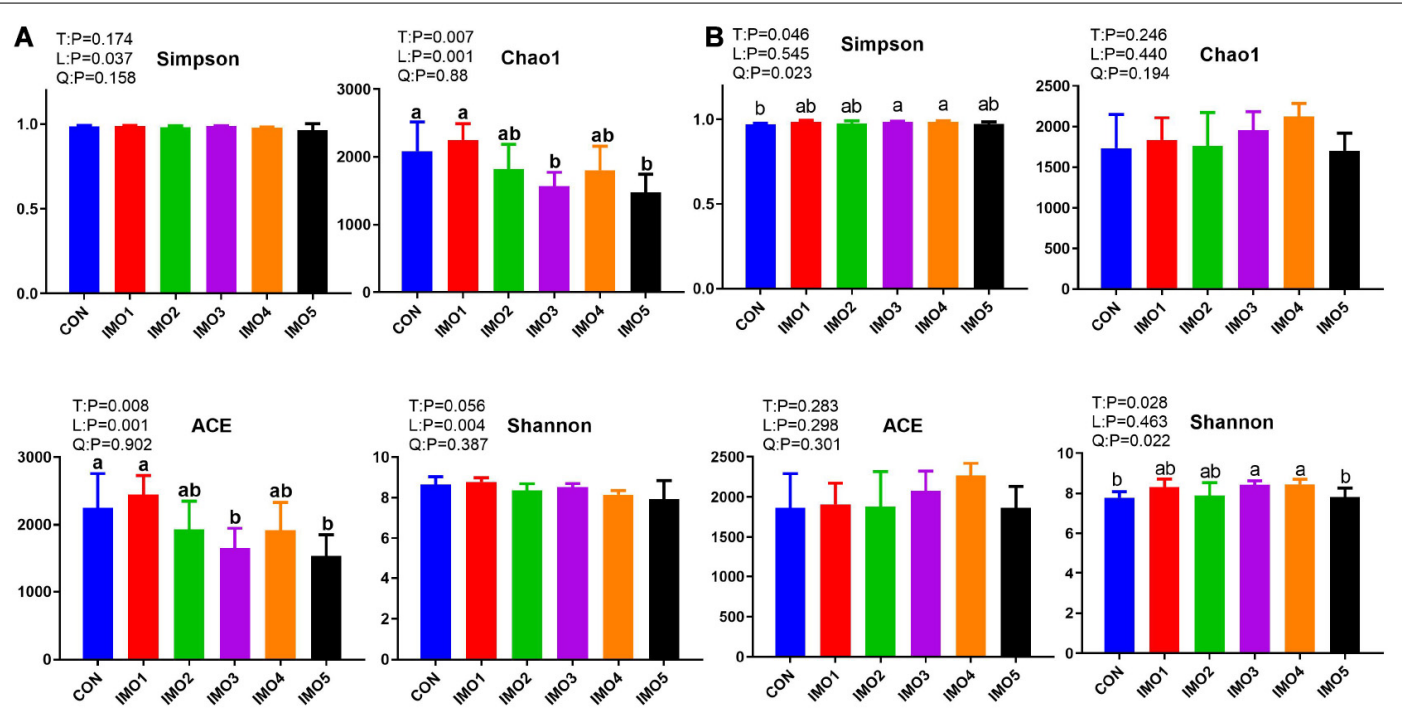

FIGURE 5 | Effects of dietary isomaltooligosaccharide (IMO) levels on the $\alpha$-diversity of gut microbiota at day 107 of gestation (A) and at day 10 of lactation (B) of sows. Simpson index, Chao1 index, ACE index, and Shannon index. Data are presented as means \pm SD $(n=5)$. (a-c) Significant effect of treatment $(P<0.05$; values with different lowercase letters are significantly different; T, total; L, linear; Q, quadratic). 

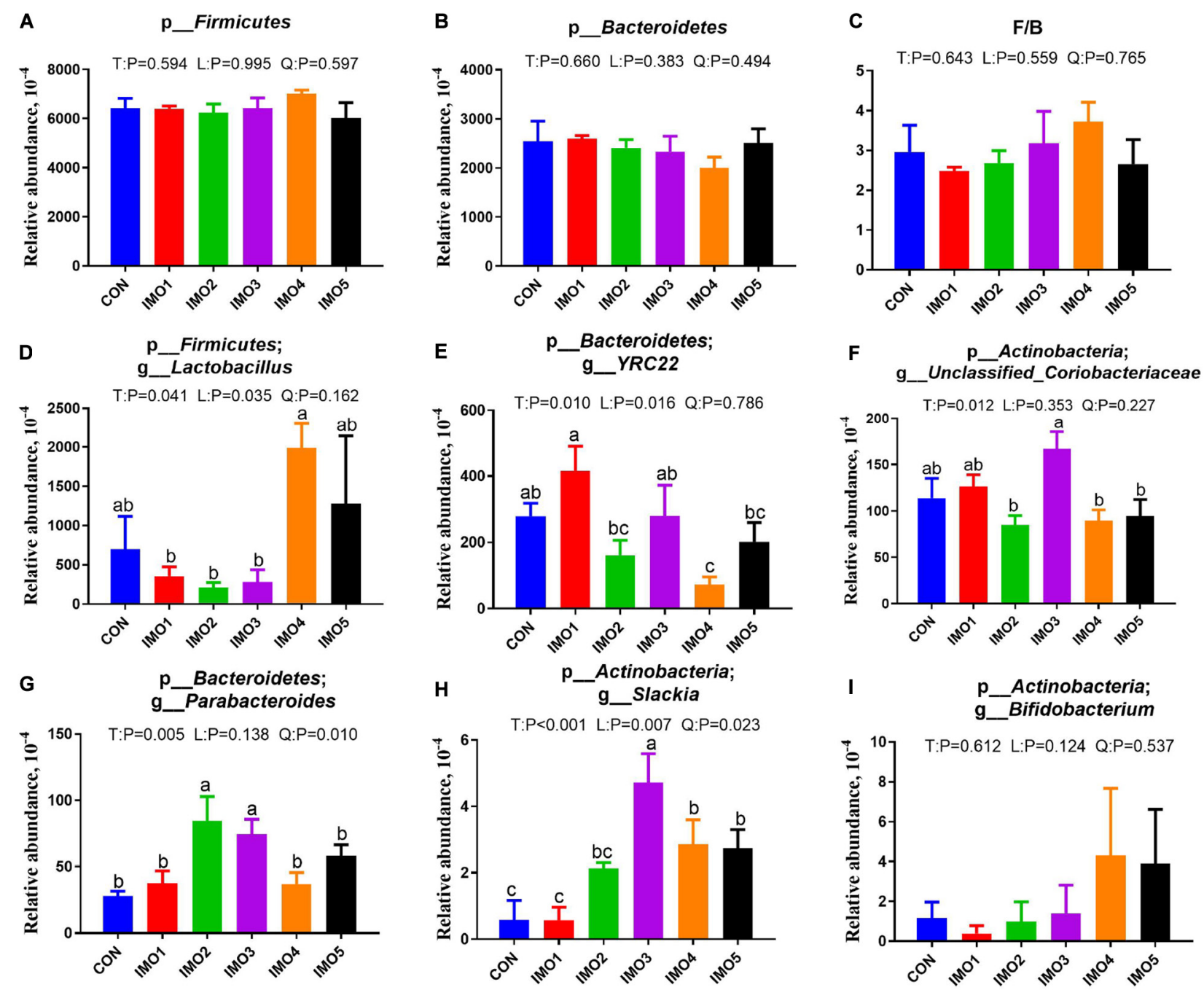

FIGURE 6 | Effects of dietary isomaltooligosaccharide (IMO) levels on the gut microbiota compositions at the phylum level in sows. (A) p_Firmicutes, (B) p_Bacteroidetes, (C) F/B, Firmicutes/Bacteroidetes, (D) p_Firmicutes; g_Lactobacillus, (E) p_Bacteroidetes;g_YRC22, (F) p_Actinobacteria;

g_Unclassified_Coriobacteriaceae, (G) p_Bacteroidetes; g_Parabacteroides, (H) p_Actinobacteria; g_Slackia, (I) p_Actinobacteria; g_Bifidobacterium. Data are presented as means \pm SEM $(n=5)$. (a-c) Significant effect of treatment $(P<0.05$; values with different lowercase letters are significantly different; $T$, total; L, linear; Q, quadratic).

\section{DISCUSSION}

The health of sow plays a crucial role in the pig production, and sows' reproductive performance is an important index of economic benefit of pig farm. However, sows in the late gestation due to rapid fetal growth caused by insulin resistance and the body's metabolic syndrome will lead to gut microbiota disorder, which will affect the reproductive performance of the sows and the growth and health of their offspring (Tan et al., 2016; Cheng et al., 2020). IMO is one of the oligosaccharides which produced by enzymatic conversion of starch and widely used in the food and feed industry, which has a wide spectrum of biological activities (Ketabi et al., 2011). Previous studies have shown that dietary oligosaccharides supplementation in sows improves sows' performance by regulating the homeostasis of gut microbiota (Li et al., 2012; Duan et al., 2019). Contrary to these results, our current study showed that IMO supplementation exerted no effects on the mainly reproductive performance of sows. This discrepancy could be attributed to the time phase of IMO supplementation and different polymerization degree of commercial IMO (Hu et al., 2013).

Interestingly, we found that dietary IMO supplementation in sows significantly reduced the diarrhea rate of piglets during lactation, especially for low dose. Consistently, Wang et al. (2016) reported that the diarrhea rate of piglets linearly declined as the IMO level increased, and the beneficial effects may be due to enhanced immune status of pigs. During lactation, milk is one of the most important ways that the sows connect with its offspring (Wu et al., 2020). Moreover, the breast milk is the only source of the energy and immunity 


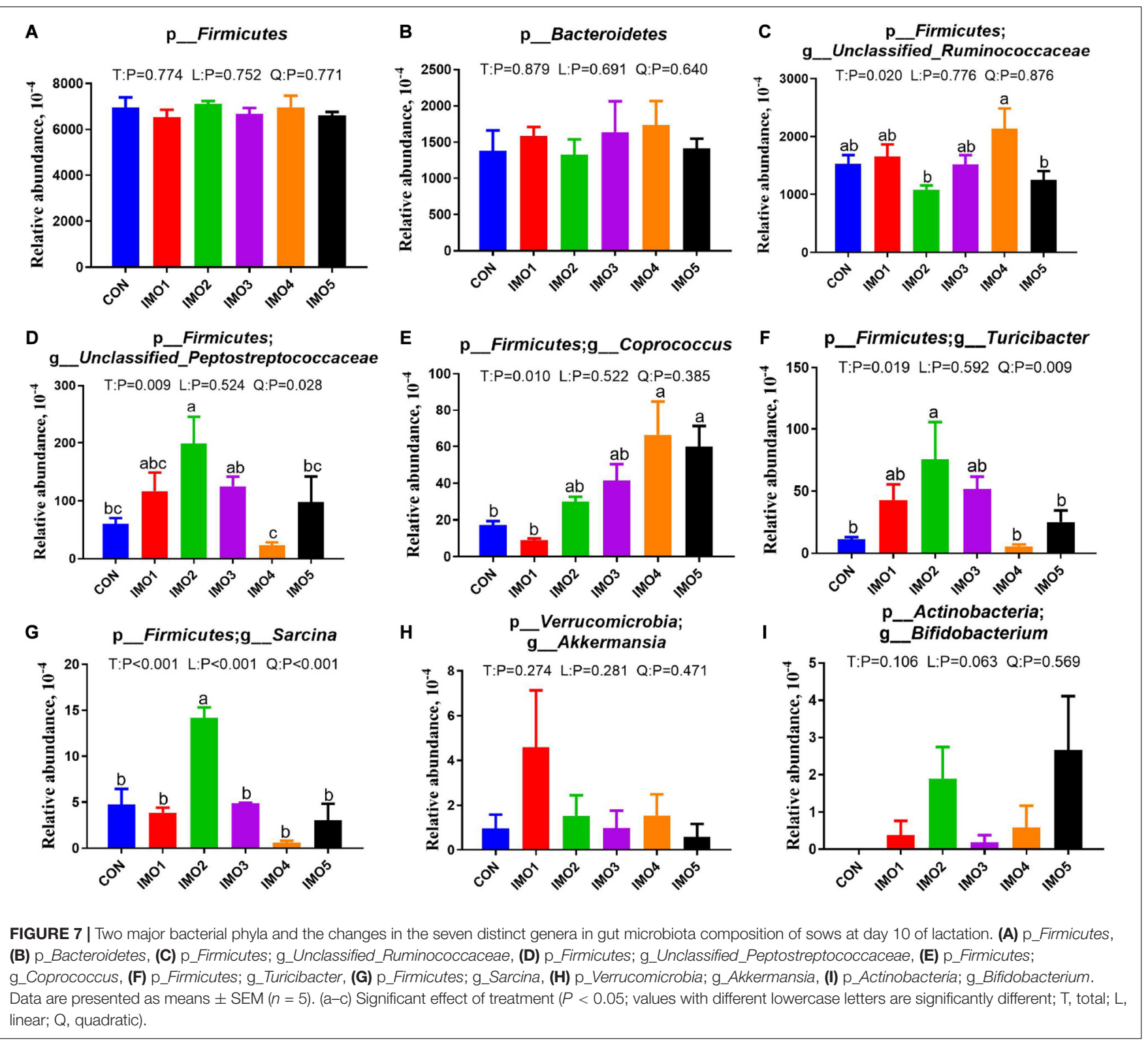

of the piglets (Xie et al., 2015), which contributes to immune system maturation, organ development, and healthy microbial colonization (Parigi et al., 2015). Many studies have showed that the milk composition and its immuneglobulin concentration play key roles in the healthy growth and development of piglets (Lonnroth et al., 1988; Charneca et al., 2015), which is closely related to the gut microbiota (Solís et al., 2010; Burton et al., 2017) and may affect the growth and development of the piglets (Kirmiz et al., 2018). A recent study indicated that chitooligosaccharides supplementation modified milk composition (Cheng et al., 2015). Similarly, previous studies have also shown that the sows fed mannanoligosaccharides improved the colostrum and milk IgA IgG, IgM, and the serum IgG level in the suckling piglets (Newman and Newman, 2001). Especially during early lactation, the diarrhea of piglets occurred due to the colostrum immunoglobulin. Therefore, we measured the colostrum composition and immunoglobulin (IgA, IgG, and $\operatorname{IgM}$ ) of sows. We found that IMO supplementation did not affect colostrum composition of sows, but improved the concentrations of colostrum IgA, IgG, and IgM, which may explain why the diarrhea rate of piglets decreased.

In addition, the injury of gut barrier function is also closely related to the milk quality. Perez et al. (2007) found that intestinally derived bacterial components are transported to the lactating breast within mononuclear cells, which affected the milk quality and the offspring health. Bacterial translocation usually caused by the destruction of gut barrier (MacFie, 2000). The concentrations of serum LPS and D-LA can be used to give expression to the gut barrier function, and if its concentration improved in serum, the gut barrier have been 


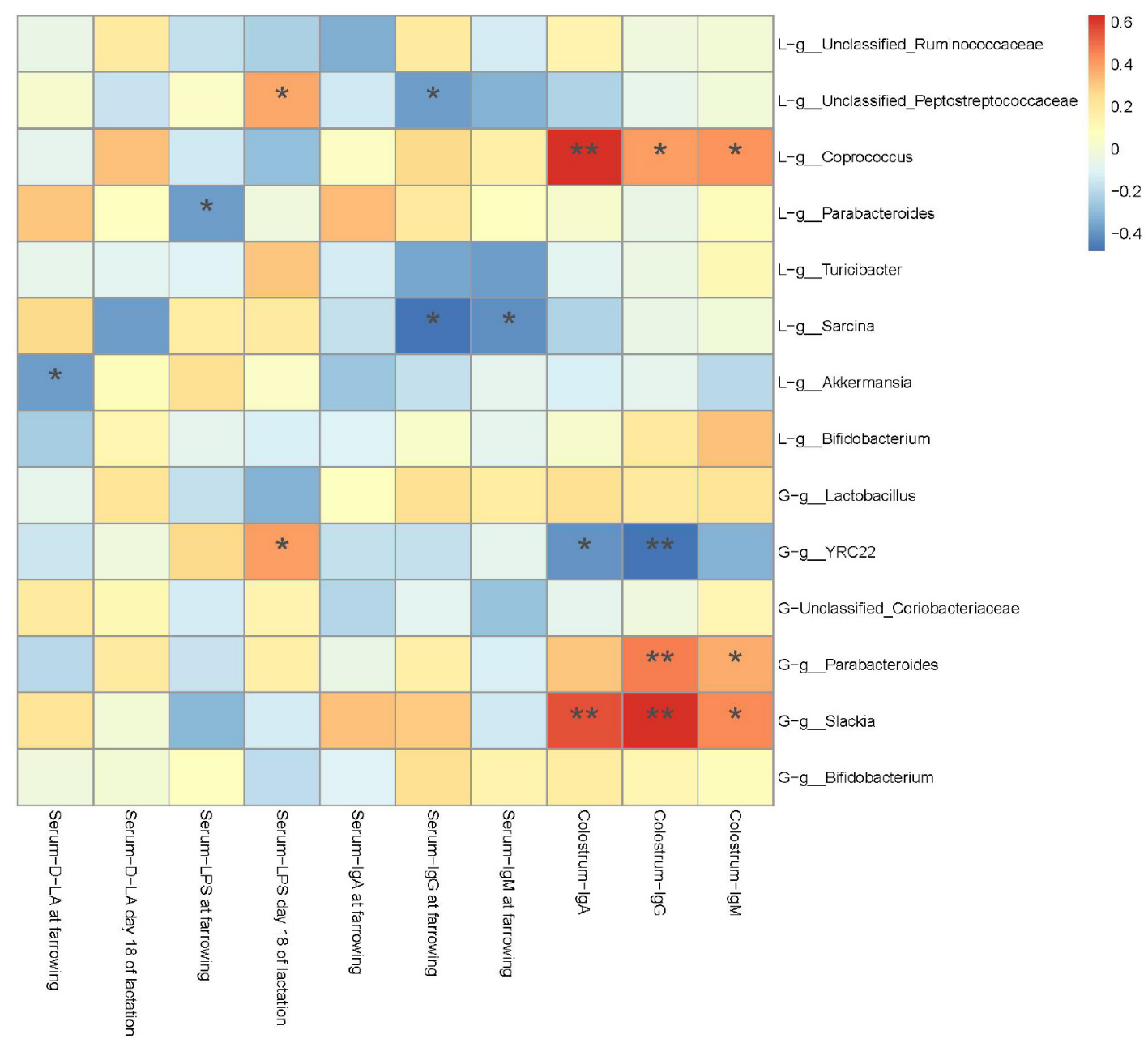

FIGURE 8 | Heatmap of the Spearman's $r$ correlations between the gut microbiota significantly modified by different dietary treatments and period of sows. Data are presented as means \pm SEM ( $n=5$ or 7 ). ${ }^{\star} P<0.05$; ${ }^{\star *} P<0.01$ (following the Spearman's correlation analysis). G, at day 107 of gestation; L, at day 10 of lactation.

destroyed (Xu et al., 2018). In the present study, the diets of sows containing IMO especially at low and high doses have lower concentrations of serum LPS and D-LA at farrowing and L18, which reflected that the function of IMO protected the intestinal barriers.

The gut microbiota is one of the most important intestinal barriers, whose homeostasis will also affect the body immunity, including the milk immunity (Rooks and Garrett, 2016). As we know, the level of immunoglobulin expression in serum of sows can immediately reflect the body immunity, which will also cause the changes of gut bacteria. In the present study, we found that IMO with different doses changed the concentrations of serum IgA, IgG, and IgM, especially IMO5 was very significant increased the immunoglobulin expression in serum of sows. To some extent, this suggests that IMO can increase the immunity of perinatal sows, which influence the structure of gut microbiota in sows. Furthermore, the establishment of gut microbiota of piglets was influenced by sow's gut microbiota, which has already been demonstrated after a probiotic treatment of mother sows (Mori et al., 2011; Baker et al., 2013; Starke et al., 2013). Recent studies have suggested that diarrhea is strongly related to the dysbiosis of gut microbiota (Shrivastava et al., 2017; The et al., 2018). In addition, the disorderliness of gut microbiota during perinatal period of sows will affect its milk quality and gut microbiota of piglets, which may lead to diarrhea in piglets. Therefore, the functional pro(e)biotic supplementation to the diets of sows during perinatal period may improve the diarrhea of piglets by regulating sow's gut microbiota.

In the present study, we found that IMO with different doses changed the composition and structure of the gut microbiota of perinatal sows, especially G107 and L10. Except for the numbers of g_YRC22, the bacterial groups in the feces of the sows were increased with varying degrees by the dietary inclusion of IMO. In G107, dietary supplementation of IMO increased the abundance of g_Parabacteroides, belonging to p_Bacteroidetes, which were comprises anti-inflammatory bacteria (Ishioka et al., 2017). The relative abundance of bacterial g_Slackia, belonging to p_Actinobacteria, was also increased. It can produce some 
bioactive substances to play a protective role and correlated with immunity. The same as our result, g_Parabacteroides and g_Slackia were closely correlated with colostrum IgA, IgG, and IgM, which may influence the diarrhea of piglets by this way.

Moreover, dietary supplementation of IMO in L10 can also significantly enhanced the relative abundance of bacterial g_Unclassified_Peptostreptococcaceae, g_Coprococcus, g_Turicibacter and g_Sarcina, which both belong to the p_Firmicutes. g_Unclassified_Peptostreptococcaceae was the dominant bacteria genus to produce the volatile fatty acids (VFAs), which contributed to the energy metabolism of host ( $\mathrm{Li}$ et al., 2018). g_Coprococcus have been found to play beneficial roles in maintaining intestinal stability, also reported as the butyrate-producing genus (Nishino et al., 2018). g_Turicibacter and g_Sarcina are also in connection with intestinal barrier function and inflammation (Liu et al., 2014; Le Sciellour et al., 2019). It systematically enhancing effect of dietary IMO on the numbers of beneficial bacterium in sows can be assumed.

\section{CONCLUSION}

In conclusion, IMO could reduce the diarrhea rate of their offspring, the effects might be attributed to the enhanced immune system of piglets. This suggests that the reduced rate of diarrhea in piglets is mainly because of the role of immunoglobulin in the sows' milk, although the IMO still had a significant influence in sows' gut microbiota, which was significantly correlated with immunoglobulin in milk. In our studies, it has evaluated the potential of IMO with regard to the sow-piglet-axis, but the concrete mechanism needs to be further researched.

\section{DATA AVAILABILITY STATEMENT}

The original contributions presented in the study are publicly available. This data can be found here: https://www.ncbi.nlm.nih. gov/bioproject/PRJNA681971.

\section{REFERENCES}

Baker, A. A., Davis, E., Spencer, J. D., Moser, R., and Rehberger, T. (2013). The effect of a bacillus-based direct-fed microbial supplemented to sows on the gastrointestinal microbiota of their neonatal piglets. J. Anim. Sci. 91, 3390-3399. doi: $10.2527 /$ jas.2012-5821

Burton, K. J., Rosikiewicz, M., Pimentel, G., Bütikofer, U., von Ah, U., et al. (2017). Probiotic yogurt and acidified milk similarly reduce postprandial inflammation and both alter the gut microbiota of healthy, young men. Br. J. Nutr. 117, 1312-1322. doi: 10.1017/S000711451700 0885

Caporaso, J. G., Kuczynski, J., Stombaugh, J., Bittinger, K., Bushman, F. D., Costello, E. K., et al. (2010). Qiime allows analysis of high-throughput community sequencing data. Nat. Methods 7, 335-336. doi: 10.1038/nmeth. f.303

Charneca, R., Vila-Viçosa, M. J., Infante, P., Nunes, J., and Le Dividich, J. (2015). Colostrum production of alentejano and large-white $\times$ landrace sows:

\section{ETHICS STATEMENT}

The animal study was reviewed and approved by the National Institutes of Health (Changsha, China) guidelines for the care and use of experimental animals.

\section{AUTHOR CONTRIBUTIONS}

ZYF, ZHS, and XH conceived and designed the experiment. LLZ, XLG, JW, and SL performed the experiments. LLZ, XLG, and $\mathrm{HL}$ analyzed the data and wrote the manuscript. YHD and ZYF revised the manuscript. All authors contributed to the article and approved the submitted version.

\section{FUNDING}

This study was jointly supported by funding from National Natural Science Foundation of China (U20A2054), the National Key R\&D Program of Intergovernmental Key Projects of China (2018YFE0101700), Double first-class construction project of Hunan Agricultural University (SYL201802015 and SYL201802009), and Support project for Scientific and Technical Talents in Hunan Province (2020TJ-Q02).

\section{ACKNOWLEDGMENTS}

The authors extend their gratitude to the staff of Hunan Agricultural University and Hunan Xinguangan Agriculture and Animal Husbandry Co., Ltd., Pingjiang Branch (Xinguangan, Inc., Hunan, China) and to their graduate students for their assistance in this study.

\section{SUPPLEMENTARY MATERIAL}

The Supplementary Material for this article can be found online at: https://www.frontiersin.org/articles/10.3389/fmicb. 2020.588986/full\#supplementary-material

consumption, passive immunity and mortality of piglets. Spanish J. Agric. Res. 13, e611. doi: 10.5424/sjar/2015134-7537

Cheng, C., Wu, X., Zhang, X., Zhang, X., and Peng, J. (2020). Obesity of sows at late pregnancy aggravates metabolic disorder of perinatal sows and affects performance and intestinal health of piglets. Animals 10, 49. doi: 10.3390/ ani10010049

Cheng, L. K., Wang, L. X., Xu, Q. S., Huang, L. J., Zhou, D. S., Li, Z., et al. (2015). Chitooligosaccharide supplementation improves the reproductive performance and milk composition of sows. Livest. Sci. 174, 74-81. doi: 10.1016/j.livsci.2015. 02.003

Duan, X., Tian, G., Chen, D., Huang, L., Zhang, D., Zheng, P., et al. (2019). Mannan oligosaccharide supplementation in diets of sow and (or) their offspring improved immunity and regulated intestinal bacteria in piglet1. J. Anim. Sci. 97, 4548-4556. doi: 10.1093/jas/ skz318

Gao, L. M., Liu, Y. L., Zhou, X., Zhang, Y., Wu, X., and Yin, Y. L. (2020). Maternal supplementation with uridine influences fatty acid and amino acid constituents 
of offspring in a sow-piglet model. Br. J. Nutr. doi: 10.1017/S0007114520003165 [Epub ahead of print].

Grześkowiak, A., Teske, J., Zentek, J., and Vahjen, W. (2020). Distinct patterns of microbial metabolic fingerprints in sows and their offspring: a pilot study. Arch. Microbiol. 202, 511-517. doi: 10.1007/s00203-019-01766-1

Hu, Y., Ketabi, A., Buchko, A., and Ganzle, M. G. (2013). Metabolism of isomalto-oligosaccharides by Lactobacillus reuteri and bifidobacteria. Lett. Appl. Microbiol. 57, 108-114. doi: 10.1111/lam.12076

Ishioka, M., Miura, K., Minami, S., Shimura, Y., and Ohnishi, H. (2017). Altered gut microbiota composition and immune response in experimental steatohepatitis mouse models. Digest. Dis. Sci. 62, 396-406. doi: 10.1007/s10620-016-4393-x

Ketabi, A., Dieleman, L. A., and Ganzle, M. G. (2011). Influence of isomaltooligosaccharides on intestinal microbiota in rats. J. Appl. Microbiol. 110, 1297-1306. doi: 10.1111/j.1365-2672.2011.04984.x

Kirmiz, N., Robinson, R. C., Shah, I. M., Barile, D., and Mills, D. A. (2018). Milk glycans and their interaction with the infant-gut microbiota. Annu. Rev. Food Sci. Technol. 9, 429-450. doi: 10.1146/annurev-food-030216-030207

Klobasa, F., Werhahn, E., and Butler, J. E. (1987). Composition of sow milk during lactation. J. Anim. Sci. 64, 1458-1466. doi: 10.2527/jas1987.6451458x

Le Sciellour, M., Zemb, O., Hochu, I., Riquet, J., Gilbert, H., Giorgi, M., et al. (2019). Effect of chronic and acute heat challenges on fecal microbiota composition, production, and thermoregulation traits in growing pigs1,2. J. Anim. Sci. 97, 3845-3858. doi: 10.1093/jas/skz222

Li, M., Bauer, L. L., Chen, X., Wang, M., Kuhlenschmidt, T. B., Kuhlenschmidt, M. S., et al. (2012). Microbial composition and in vitro fermentation patterns of human milk oligosaccharides and prebiotics differ between formula-fed and sow-reared piglets. J. Nutr. 142, 681-689. doi: 10.3945/jn.111.154427

Li, X., Liu, G., Liu, S., Ma, K., and Meng, L. (2018). The relationship between volatile fatty acids accumulation and microbial community succession triggered by excess sludge alkaline fermentation. J. Environ. Manag. 223, 85-91. doi: 10.1016/j.jenvman.2018.06.002

Likotrafiti, E., Tuohy, K. M., Gibson, G. R., and Rastall, R. A. (2014). An in vitro study of the effect of probiotics, prebiotics and synbiotics on the elderly faecal microbiota. Anaerobe 27, 50-55. doi: 10.1016/j.anaerobe.2014.03.009

Liu, J., Xu, T., Zhu, W., and Mao, S. (2014). High-grain feeding alters caecal bacterial microbiota composition and fermentation and results in caecal mucosal injury in goats. Br. J. Nutr. 112, 416-427. doi: 10.1017/ S0007114514000993

Lonnroth, I., Martinsson, K., and Lange, S. (1988). Evidence of protection against diarrhoea in suckling piglets by a hormone-like protein in the sow's milk. Zentralbl Veterinarmed B 35, 628-635. doi: 10.1111/j.1439-0450.1988.tb0 0537.x

MacFie, J. (2000). Enteral versus parenteral nutrition: the significance of bacterial translocation and gut-barrier function. Nutrition 16, 606-611. doi: 10.1016/ s0899-9007(00)00249-5

Mori, K., Ito, T., Miyamoto, H., Ozawa, M., Wada, S., Kumagai, Y., et al. (2011). Oral administration of multispecies microbial supplements to sows influences the composition of gut microbiota and fecal organic acids in their post-weaned piglets. J. Biosci. Bioeng. 112, 145-150. doi: 10.1016/j.jbiosc.2011.04.009

Newman, K. E., and Newman, K. C. (2001). Evaluation of mannanoligosaccharides on the microflora and immunoglobulin status of sows and piglet performance. J. Anim. Sci. Biotechnol. 79, 189. doi: 10.1186/s40104-020-00480-z

Nishino, K., Nishida, A., Inoue, R., Kawada, Y., Ohno, M., Sakai, S., et al. (2018). Analysis of endoscopic brush samples identified mucosa-associated dysbiosis in inflammatory bowel disease. J. Gastroenterol. 53, 95-106. doi: 10.1007/s00535017-1384-4

Parigi, S. M., Eldh, M., Larssen, P., Gabrielsson, S., and Villablanca, E. J. (2015). Breast milk and solid food shaping intestinal immunity. Front. Immunol. 6:415. doi: $10.3389 /$ fimmu.2015.00415

Perez, P. F., Dore, J., Leclerc, M., Levenez, F., Benyacoub, J., Serrant, P., et al. (2007). Bacterial imprinting of the neonatal immune system: lessons from maternal cells? Pediatrics 119, e724-e732. doi: 10.1542/peds.2006-1649

Rooks, M. G., and Garrett, W. S. (2016). Gut microbiota, metabolites and host immunity. Nat. Rev. Immunol. 16, 341-352. doi: 10.1038/nri.2016.42

Salmon, H., Berri, M., Gerdts, V., and Meurens, F. (2009). Humoral and cellular factors of maternal immunity in swine. Dev. Comp. Immunol. 33, 384-393. doi: 10.1016/j.dci.2008.07.007
Shrivastava, A. K., Kumar, S., Mohakud, N. K., Suar, M., and Sahu, P. S. (2017). Multiple etiologies of infectious diarrhea and concurrent infections in a pediatric outpatient-based screening study in Odisha, India. Gut Pathog. 9, 16. doi: 10.1186/s13099-017-0166-0

Solís, G., de Los Reyes-Gavilan, C. G., Fernández, N., Margolles, A., and Gueimonde, M. (2010). Establishment and development of lactic acid bacteria and bifidobacteria microbiota in breast-milk and the infant gut. Anaerobe 16, 307-310. doi: 10.1016/j.anaerobe.2010.02.004

Starke, I. C., Pieper, R., Neumann, K., Zentek, J., and Vahjen, W. (2013). Individual responses of mother sows to a Probioticenterococcus faecium strain lead to different microbiota composition in their offspring. Beneficial Microbes 4, 345-356. doi: 10.3920/BM2013.0021

Tan, C., Wei, H., Ao, J., Long, G., and Peng, J. (2016). Inclusion of konjac flour in the gestation diet changes the gut microbiota, alleviates oxidative stress, and improves insulin sensitivity in sows. Appl. Environ. Microbiol. 82, 5899-5909. doi: 10.1128/AEM.01374-16

The, H. C., Florez, D. S. P., Jie, S., Pham, T. D., Thompson, C. N., Minh, C. N. N., et al. (2018). Assessing gut microbiota perturbations during the early phase of infectious diarrhea in vietnamese children. Gut Microbes 9, 38-54. doi: 10.1080/ 19490976.2017.1361093

Wang, X. X., Song, P. X., Wu, H., Xue, J. X., Zhong, X., and Zhang, L. Y. (2016). Effects of graded levels of isomaltooligosaccharides on the performance, immune function and intestinal status of weaned pigs. Asian Aust. J. Anim. Sci. 29, 250-256. doi: 10.5713/ajas.15.0194

Wu, X., Gao, L.-M., Liu, Y.-l, Xie, C., Cai, L., Xu, K., et al. (2020). Maternal dietary uridine supplementation reduces diarrhea incidence in piglets by regulating the intestinal mucosal barrier and cytokine profiles. J. Sci. Food Agric. 100, 3709-3718. doi: 10.1002/jsfa.10410

Wu, Y., Pan, L., Shang, Q. H., Ma, X. K., Long, S. F., Xu, Y. T., et al. (2017). Effects of isomalto-oligosaccharides as potential prebiotics on performance, immune function and gut microbiota in weaned pigs. Anim. Feed Sci. Technol. 230, 126-135. doi: 10.1016/j.anifeedsci.2017. 05.013

Xie, C., Guo, X., Long, C., Fan, Z., Xiao, D., Ruan, Z., et al. (2015). Supplementation of the sow diet with chitosan oligosaccharide during late gestation and lactation affects hepatic gluconeogenesis of suckling piglets. Anim. Reprod. Sci. 159, 109-117. doi: 10.1016/j.anireprosci.2015. 06.004

Xu, J., Liu, Z., Zhan, W., Jiang, R., Yang, C., Zhan, H., et al. (2018). Recombinant tsp53 modulates intestinal epithelial barrier integrity via upregulation of zol in lpsinduced septic mice. Mol. Med. Rep. 17, 1212-1218. doi: 10.3892/mmr.2017. 7946

Yen, C., Tseng, Y., Kuo, Y., Lee, M., and Chen, H. (2011). Long-term supplementation of isomalto-oligosaccharides improved colonic microflora profile, bowel function, and blood cholesterol levels in constipated elderly people-a placebo-controlled, diet-controlled trial. Nutrition 27, 445-450. doi: 10.1016/j.nut.2010.05.012

Zambrano, E., Ibáñez, C., Martínez-Samayoa, P. M., Lomas-Soria, C., DurandCarbajal, M., Rodríguez-González, G. L., et al. (2016). Maternal obesity: lifelong metabolic outcomes for offspring from poor developmental trajectories during the perinatal period. Arch. Med. Res. 47, 1-12. doi: 10.1016/j.arcmed.2016.01. 004

Conflict of Interest: The authors declare that the research was conducted in the absence of any commercial or financial relationships that could be construed as a potential conflict of interest.

The reviewer XX declared a shared affiliation with one of the authors YHD, to the handling editor at the time of the review.

Copyright $\odot 2021$ Zhang, Gu, Wang, Liao, Duan, Li, Song, He and Fan. This is an open-access article distributed under the terms of the Creative Commons Attribution License (CC BY). The use, distribution or reproduction in other forums is permitted, provided the original author(s) and the copyright owner(s) are credited and that the original publication in this journal is cited, in accordance with accepted academic practice. No use, distribution or reproduction is permitted which does not comply with these terms. 